\title{
Explorando la relación de la adicción a la compra con otros comportamientos excesivos: un estudio piloto
}

\author{
Rafael Rodríguez-Villarino*; Manuel González-Lorenzo**; Ángel Fernández-González***; \\ María Lameiras-FernándeZ** \\ *Profesor-Tutor. Departamento de Métodos de Investigación y Diagnóstico en Educación II (MIDE-II) (Orientación Educativa, \\ Diagnóstico e Intervención Psicopedagógica). U.N.E.D. (Centro Asociado de Ourense). \\ * * Profesor Titular. Departamento de Análisis e Intervención Psicosocioeducativa. Universidad de Vigo. \\ ***Profesor Asociado. Departamento de Análisis e Intervención Psicosocioeducativa. Universidad de Vigo.
}

\section{RESUMEN}

Este artículo explora, utilizando una muestra de adultos urbanos de ambos sexos ( $n=821 ; 393$ varones y 428 mujeres), la relación de la adicción a la compra con la frecuencia percibida de realización de cinco comportamientos: comer en exceso, fumar en exceso, jugar en exceso, ingerir bebidas alcohólicas de modo problemático y consumir otras sustancias psicoactivas (p. ej., marihuana, éxtasis, etc.). Los hallazgos sugieren que la adicción a la compra covaría con las mencionadas conductas excesivas conformando, en conjunto, un factor general de "adictividad", en cuya estructura jerárquica ocupa un lugar "intermedio" con respecto a los restantes excesos. Estos resultados se discuten en relación a la comunalidad entre adicciones químicas y conductuales, presentándose sugerencias referidas a la investigación e intervención sobre comportamientos adictivos.

Palabras-clave: adicción a la compra, juego excesivo, consumo problemático de bebidas alcohólicas, comer en exceso, personalidad adictiva, conductas adictivas múltiples.

\begin{abstract}
Using a sample of urban adults of both sexes ( $n=821$; 393 men and 428 women), this article explores the association of shopping addiction with the perceived frequency of five behaviours: eating in excess, smoking in excess, gambling in excess, problematic alcohol use and taking other psychoactive substances (i.e., marijuana, ecstasy, etc.). The findings suggest that buying addiction covaries with the abovementioned excessive behaviours shaping, as a whole, a general factor of "addictiveness", in whose hierarchical structure it occupies an "intermediate" place in comparison with the other excesses. These results are discussed in relation to the commonality between chemical and behavioural addictions, presenting suggestions related to research and intervention in addictive behaviours.
\end{abstract}

Keywords: buying addiction, gambling in excess, problematic alcohol use, eating in excess, addictive personality, multiple addictive behaviours.

\section{INTRODUCCIÓN}

$\mathbf{U}$ na de las definiciones más consensuadas sobre adicción a la compra es aquella que la identifica con motivaciones de compra persistentemente asaltantes y repetitivas, las cuales se perciben usualmente como irresistibles, placenteras y/o reductoras de malestar, pero que finalmente causan perjuicios al individuo y/o a otras personas (Friese, 1998; Nataraajan y Goff, 1991; O'Guinn y Faber, 1989). El elevado endeudamiento es la consecuencia más visible de este estilo adquisitivo, y afecta a entre el $60 \%$ y el $89 \%$ de los compradores que solicitan tratamiento (Christenson, Faber, De Zwann, Raymond, Specker, Ekern, McKenzie, Crosby, Crown, Ekert, Mussell y Mitchell, 1994; McElroy, Keck, Pope, Smith y Strakowski, 1994; Schlosser, Black, Repertinger y Freet, 1994); el malestar emocional, expresado mediante niveles elevados de estrés y dificultades de adaptación (Sánchez, 1996) y/o a través de estados depresivos y sentimientos de depresión y culpabilidad post-compra (Briney, 1989; McElroy et al., 1994) constituye, junto a los problemas de relación marital y familiar (Christenson et al., 1994; Schlosser et al., 1994), otro de los resultados negativos de esta adicción conductual. Una 
adicción que tiene, a tenor de diversas estimaciones, una notoria presencia en las sociedades de consumo occidentales. De hecho, se han constatado cifras de prevalencia que varían desde alrededor del $2 \%$ hasta el 7\%, dependiendo de la zona geográfica y de la definición de compra adictiva que se ha utilizado (Faber y O’Guinn, 1992; Olábarri y García, 2003; Luna, 2002; Reisch y Scherhorn, 1996; Roberts y Martínez, 1997; Rodríguez-Villarino, 2004).

La descripción de similitudes entre la adicción a la compra y otras adicciones ha sido uno de los medios a los que se ha recurrido para reflejar el cariz adictivo de este comportamiento adquisitivo. Así, King (1981) definió el "consumo adictivo" como una conducta anormal del comprador que evidencia una sintomatología similar a la que está presente en otras formas de consumo patológico (alcoholismo, toxicomanía, bulimia, etc.): deseo obsesivo, compulsión por comprar, pérdida de control conductual, desarrollo de tolerancia (tendencia a incrementar la conducta de compra) y aparición de dependencia psíquica. Estas características son manifiestas en el estudio de caso realizado por Glatt y Cook en 1987, y condujeron a que estos investigadores señalasen la conveniencia de considerar el "gasto patológico" como un tipo de dependencia psicológica, lo cual también ha sido subrayado por otros autores (p. ej., Faber, O'Guinn y Krych, 1987; Reisch y Scherhorn, 1996). Asimismo, no faltan quienes postulan que la conducta de compra adictiva se rige por el modelo prototípico de ciclo adictivo: malestar emocional $\rightarrow$ compra $\rightarrow$ consecuencias positivas $\rightarrow$ consecuencias negativas $\rightarrow$ malestar emocional $\rightarrow$ compra (p. ej., Edwards, 1992; Faber y O'Guinn, 1989).

Además de estos posicionamientos teóricos, la comunalidad de la adicción a la compra con otras adicciones se ha analizado, fundamentalmente, a través de estudios de comorbilidad realizados con muestras de adictos a la compra "crónicos". En estos trabajos se encontraron porcentajes elevados de personas que presentan, en algún momento de sus vidas -prevalencia vital-, diagnósticos de trastorno por consumo de sustancias psicoactivas (especialmente abuso y dependencia del alcohol), trastornos de la alimentación, juego patológico, otros trastornos del control de impulsos y adicción al sexo (p. ej., Black, Repertinger, Gaffney y Gabel, 1998; Christenson et al., 1994; McEIroy et al., 1994; Schlosser et al., 1994). De modo más específico, Faber, Christenson, De Zwann y Mitchell (1995) encontraron que el riesgo de adicción a la compra es mayor en los casos con diagnósticos de trastornos alimentarios (bulimia y trastorno por atracón) y viceversa. Otro ejemplo del "nexo" de la adicción a la compra con otros excesos es patente en la investigación de Specker, Carlson, Christenson y Marcotte (1995): los autores hallaron que la adicción a la compra y la adicción al sexo son significativamente más elevadas en un grupo de jugadores patológicos en tra- tamiento que en un grupo de control (la adicción a la compra estaba presente en el $25 \%$ de los jugadores patológicos y la adicción al sexo en el 10\% de éstos).

En resumen, los hallazgos encontrados en los estudios de comorbilidad aportan evidencias de que la adicción a la compra se relaciona con problemas de alcohol, tabaco y otras drogas, de alimentación (bulimia y trastorno por atracón, sobre todo) y con algunos trastornos del control de impulsos (juego patológico, cleptomanía, trastorno explosivo intermitente y adicción al sexo). No obstante, estos resultados proceden de trabajos llevados a cabo con muestras de adictos a la compra -o de otros adictos- de tipo clínico y tamaño reducido, lo cual limita la posibilidad de generalizarlos.

Una alternativa a estos acercamientos la constituyen las investigaciones que examinan la relación de la adicción a la compra con otras adicciones en la población general. La realizada por Roberts y Tanner en 2000 es la única, al menos en nuestro conocimiento, que ha seguido esta orientación. Estos investigadores utilizaron una muestra de 111 adolescentes escolarizados, y hallaron que la adicción a la compra covaría con el consumo de tabaco $(r=.30)$, alcohol $(r=.31)$ y drogas (.32), así como con la intención de tener relaciones sexuales precoces $(r=.30)$. Además, Roberts y Tanner encontraron correlaciones significativas entre estas "conductas e intenciones de riesgo", lo cual sugiere, a su juicio, que pueden compartir una dimensión común. El presente estudio se enmarca en esta perspectiva de análisis y examinará la asociación de la adicción a la compra con diversos excesos, para lo cual empleará una muestra de adultos de la población general como grupo de estudio.

\section{MÉTODO}

Esta investigación es de carácter exploratorio y recurre a medidas de autoinforme para analizar la relación entre adicción a la compra y frecuencia percibida de realización de varios excesos. Las informaciones recabadas con respecto a esta materia forman parte de un trabajo más amplio sobre adicción a la compra -temática que se "enmascaró" como un estudio sobre "calidad de vida"- y se recogieron a través de cuestionarios administrados en los domicilios por encuestadores debidamente entrenados.

\section{Muestra}

El grupo utilizado en esta investigación está conformado por adultos de ambos sexos, los cuales pro- 
vienen de las cuatro capitales de provincia gallegas ( $A$ Coruña, Lugo, Ourense y Pontevedra) y de la capital administrativa de esta Comunidad Autónoma (Santiago de Compostela). Con el objeto de alcanzar la representatividad muestral, se distribuyeron los cuestionarios puerta-a-puerta siguiendo un procedimiento de rutas aleatorias, administrándose 200 en todas las ciudades excepto en Lugo, donde el número se redujo a 100. El proceso se inició en diciembre de 2000 y finalizó en septiembre de 2002. Puesto que la adicción a la compra es la materia de estudio fundamental, se decidió emplear una muestra con suficiente capacidad económica para desarrollar este comportamiento adictivo y, por esta razón, se situó el límite inferior de edad en 25 años (momento en el que, a priori, la mayoría de las personas trabajan), limitándose el margen superior a 65 años (ya que ésta es la edad legal de jubilación en España y supone, en muchas ocasiones, una reducción de la capacidad financiera). Un análisis de deseabilidad social, realizado mediante una escala enmascarada en el cuestionario general, redujo la muestra de 900 a 821 participantes (393 varones y 428 mujeres). Este grupo final mostró una edad media de 42.1 años y una desviación típica de 12.1 años; la mayoría de las personas están casadas (57.1\%), tienen hasta educación secundaria $(67.8 \%$; el grupo más numeroso es el de estudios secundarios: $36.5 \%$ ), trabajan $(72.5 \%)$ y cuentan con niveles de ingresos entre 1200 y 1800 euros. Esta muestra representaría, con un error muestral de $\pm 3.49 \%$ (para $p=q=50 \%$ y un nivel de confianza de 2 \&\#1073), la población adulta entre 25 y 65 años de las urbes de referencia (universo: 531.066 habitantes), si bien la existencia de un $28 \%$ de negativas en el momento de cumplimentar el cuestionario aconseja mantener cierta cautela con respecto a esta representatividad.

\section{Medidas}

En el presente trabajo utilizamos medidas de autoinforme para evaluar la adicción a la compra y la frecuencia percibida de realización excesiva de cinco conductas (comer, fumar, jugar a juegos de azar, consumir bebidas alcohólicas y usar drogas).

La adicción a la compra se evalúo mediante el German Addictive Buying Indicator (GABI; Scherhorn et al., 1990). Este indicador consta de 16 ítems, debiendo señalar la persona su nivel de acuerdo (escala de cuatro puntos; desde totalmente en desacuerdo -valor 1- a totalmente de acuerdo -valor 4-) con respecto a los diferentes enunciados (ver apéndice 1). Scherhorn et al. recurrieron a un grupo de adictos a la compra crónicos ( $n=26$ ) y a un grupo de participantes de la población general ( $n=138$ ) para examinar las propiedades psicométricas -fiabilidad, estructura factorial y validez- del GABI. Los autores constataron que esta medida presenta un nivel satisfactorio de consistencia interna en el grupo de adictos (alfa de Cronbach $=.87$ ), el cual se incrementa al combinarlos con el grupo de compradores "normales" (alcanzando un alfa de .92). Los resultados del análisis factorial con rotación varimax evidenciaron, también en ambos grupos, que el GABI es una medida de naturaleza básicamente unidimensional. Estos investigadores alemanes corroboraron la validez de constructo de su escala al confirmar, con independencia de la muestra empleada, la hipotética relación de ésta con tres variables de personalidad: positiva con obsesión-compulsión y depresión, y negativa con autoestima. Por último, Scherhorn y sus colaboradores encontraron que la puntuación media del grupo de adictos en el GABI es significativamente superior a la del grupo de la población general (45 vs. $27 ; p<.01$ ), lo cual sugiere que esta herramienta posee, empleando sus propios términos, "validez del grupo extremo". Rodríguez (2004) examinó la consistencia interna y la estructura factorial del GABI en un grupo de adultos gallegos de ambos sexos ( $n=821$ ). Los resultados de esta investigación evidencian que la escala en cuestión presenta un alfa de Cronbach muy elevado (.92); en cuanto a dimensionalidad, el análisis de componentes principales mostró una solución de tres factores, si bien la relevancia explicativa del primero es muy superior (da cuenta del $48.66 \%$ de la varianza, mientras que los otros dos explican, respectivamente, el $6.89 \%$ y el $6.64 \%$ de ésta). Parece, entonces, que el instrumento de medida de la adicción a la compra construido por Scherhorn et al. tiene una notable consistencia interna y un carácter fundamentalmente unidimensional, aparentando validez con respecto al constructo que pretende evaluar (al menos según se desprende del estudio que llevaron a cabo estos investigadores en Alemania).

Utilizamos medidas de frecuencia para examinar la periodicidad percibida de realización de los restantes excesos. En concreto, solicitamos a los encuestados que indicasen la frecuencia (escala con cuatro categorías: "nunca", "pocas veces", "bastantes veces" y "muy frecuentemente") con que realizaron los siguientes comportamientos durante los seis últimos meses: 1) comer en exceso, 2) fumar en exceso, 3) jugar habitualmente a juegos de azar, 4) consumir bebidas alcohólicas de modo problemático, y 5) consumir sustancias para modificar el estado de ánimo (p. ej., marihuana, éxtasis, etc.). El rango de puntuaciones varía entre un mínimo de 0 y un máximo de 3 para cada una de las actividades reseñadas.

\section{Análisis estadístico}

La relación entre adicción a la compra y los demás "excesos" se examinó de tres modos: matriz de correlaciones, análisis factorial y análisis cluster jerarqui- 
zado. El estudio de las covariaciones se empleó con la finalidad de analizar la asociación entre pares de variables, el análisis factorial para explorar los "vínculos" entre todas las variables y el análisis cluster jerarquizado para mostrar, a través de un dendrograma, la estructura de tales vínculos. Adicionalmente, se compararon, mediante ANOVA, las diferencias entre sexos con respecto a los distintos excesos. Todos los análisis estadísticos se llevaron a cabo a través del paquete estadístico SPSS versión 11.0.

\section{RESULTADOS}

La matriz de correlaciones refleja, como se evidencia al observar la Tabla 1, la existencia de notables covariaciones entre los distintos comportamientos excesivos.

A nivel general, la tabla anterior muestra que existen correlaciones positivas y estadísticamente significativas entre la frecuencia con que se fuma, come, juega,

Tabla 1. Matriz de correlaciones de las variables objeto de estudio

\begin{tabular}{lcccccc|}
\hline Variable & $\mathbf{1}$ & $\mathbf{2}$ & $\mathbf{3}$ & $\mathbf{4}$ & $\mathbf{5}$ & $\mathbf{6}$ \\
\hline $\begin{array}{l}\text { 1. Adicción a la compra. } \\
\text { 2. Frecuencia con que se come en exceso. }\end{array}$ & & & & & & \\
3. Frecuencia con que se fuma en exceso. & $.22^{* *}$ & & & & & \\
$\begin{array}{l}\text { 4. Frecuencia con que se juega en exceso. } \\
\text { 5. Frecuencia con que se consumen bebidas alcohólicas }\end{array}$ & $.40^{* *}$ & $.22^{* *}$ & $.26^{* *}$ & & \\
$\quad$ de modo problemático. & $.40^{* *}$ & $.18^{* *}$ & $.29^{* *}$ & $.64^{* *}$ & \\
$\begin{array}{l}\text { 6. Frecuencia con que se consumen otras sustancias } \\
\text { psicoactivas (p. ej., marihuana, éxtasis...). }\end{array}$ & $.32^{* *}$ & $.18^{* *}$ & $.32^{* *}$ & $.49^{* *}$ & $.59^{* *}$ \\
\hline${ }^{* *} p=.01$ & & & & & \\
\hline
\end{tabular}

consume alcohol y tabaco y la adicción a la compra. El rango de covariaciones varía entre .18 (que se refiere a las que establece la frecuencia de ingesta excesiva de alimentos con la periodicidad de consumo problemático de alcohol y el consumo de sustancias psicoactivas) y .64 (la cual se entabla entre la frecuencia de juego excesivo y la relativa al consumo problemático de bebidas alcohólicas). La compra adictiva se asocia, en primer lugar, a la frecuencia de juego excesivo y al consumo de alcohol y drogas, posteriormente a la regularidad con que se utilizan sustancias psicoactivas y, por último, al consumo excesivo de tabaco y alimen- tos. Las "vinculaciones" reseñadas sugieren que los seis comportamientos desmedidos considerados en este trabajo podrían explicarse a través de un número de factores más reducido.

Se empleó el análisis factorial para contrastar esta tesis. Los resultados encontrados a través del método de componentes principales muestran la presencia de un único factor con valor propio igual o mayor que uno, el cual explica el $45.77 \%$ de la varianza. La Tabla 2 muestra las saturaciones en el citado factor de los distintos comportamientos excesivos.

\section{Tabla 2. Saturaciones de los distintos comportamientos excesivos en el primer Factor}

Comportamientos excesivos Saturaciones

Frecuencia de consumo problemático de bebidas alcohólicas .82

Frecuencia con que se juega en exceso

Frecuencia con que se consumen sustancias para modificar el estado de ánimo

(p. ej., marihuana, éxtasis,...)

Adicción a la compra

Frecuencia con que se fuma en exceso

Frecuencia con que se come en exceso

.39 
Estos hallazgos indican la posible existencia de un factor general de consumo excesivo o de "adictividad", el cual se relaciona estrechamente con cinco de los seis comportamientos desmedidos examinados (consumo problemático de alcohol, juego excesivo, consumo de sustancias psicoactivas, adicción a la compra y fumar en exceso), siendo no desdeñable, aunque más reducida, su asociación con el referido a la ingesta excesiva de alimentos.

Recurrimos al análisis cluster para profundizar en las relaciones que se entablan entre la adición a la compra y la frecuencia percibida con que se llevan a cabo los restantes excesos. El dendrograma, que es la expresión gráfica del análisis cluster jerarquizado, muestra a través de medidas de "distancia" cómo se estructuran u ordenan las diferentes conductas desmedidas (Figura 1).

Esta figura refleja la presencia de una agrupación general de conductas excesivas y la existencia de conglomerados más específicos. En este último caso, es manifiesta la cercanía entre frecuencia de juego excesivo y consumo problemático de alcohol, así como la

\section{Figura 1. Análisis cluster jerarquizado}

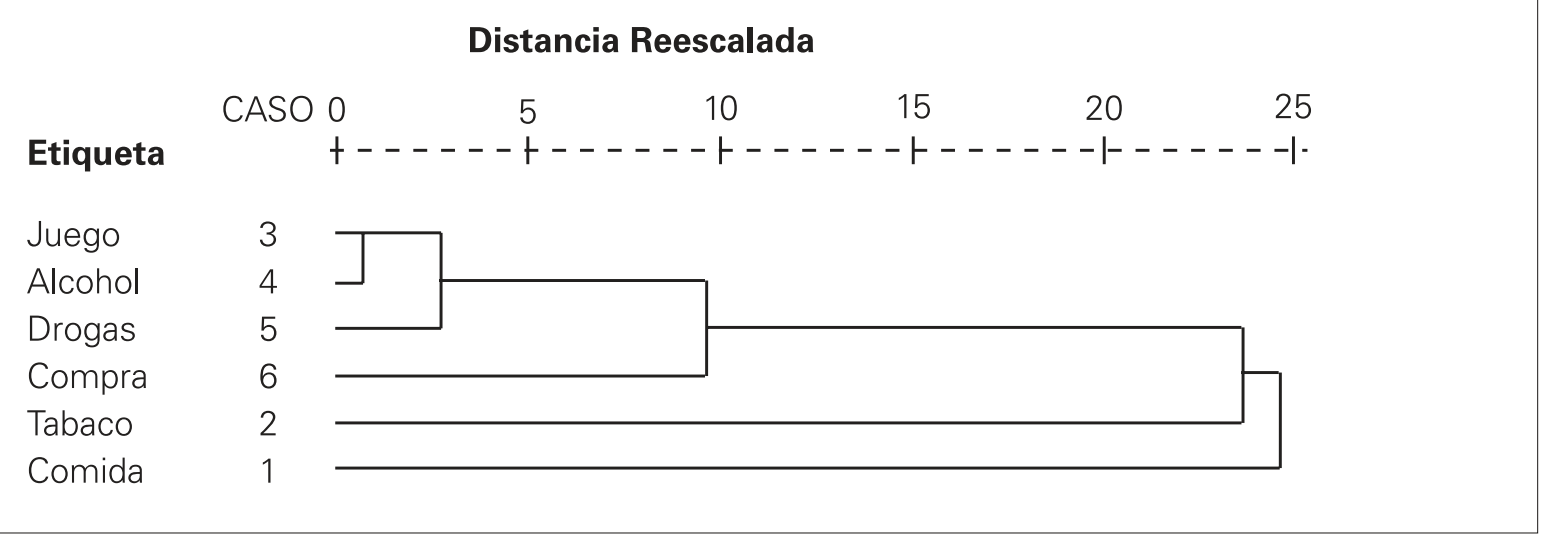

proximidad de éstos con la periodicidad en el consumo de drogas y de todos ellos con la compra adictiva. Los excesos reseñados se relacionan, aunque la separación es mayor, con la regularidad con que se consume excesivamente tabaco y, en último término, con la referida a la ingesta desmedida de alimentos. Parece, entonces, que la compra adictiva se encuentra a medio camino entre un núcleo de excesos muy relacionados (los referidos a la frecuencia de juego patológico, consumo problemático de alcohol y consumo de drogas) y la periodicidad de "otras" conductas excesivas (las relativas a fumar y comer de modo excesivo).

Una cuestión adicionalmente examinada en la presente investigación es la relativa a si los sexos se diferencian en su proclividad a diferentes conductas excesivas. Los resultados del ANOVA univariado evidencian que varones y mujeres no difieren de modo estadísticamente significativo en la frecuencia con que informan fumar o comer en exceso, pero sí en la referida a las restantes conductas desmedidas; más específicamente, se halla que las mujeres puntúan más en adicción a la compra (29.17 vs. 26.78; $p$ < .000) y los varones en frecuencia de juego excesivo (.26 vs. .19; $\mathrm{p}<.000)$, consumo problemático de alcohol (.29 vs. $.22 ; p<.001)$ y consumo de sustancias psicoactivas (.31 vs. .24); $p<.008)$.

\section{DISCUSIÓN}

Los resultados de este trabajo sugieren que la compra adictiva y la frecuencia con que se realizan "otros" comportamientos excesivos son aspectos estrechamente relacionados. Algunos de estos excesos están muy asociados entre sí (es lo que sucede en el caso del juego patológico, el consumo problemático de alcohol y el consumo de otras sustancias psicoactivas); la relación de tales excesos es también notable, aunque ligeramente inferior, con la compra adictiva, resultando de menor magnitud con respecto a la desmesura en el consumo de tabaco y la ingesta de comida.

Las relaciones entre alcohol, tabaco, juego y sustancias psicoactivas son consistentes con las encontradas en investigaciones previas. Por ejemplo, Rozin y Stoess (1993) constataron que la adicción al alcohol covaría con la adicción al juego y al tabaco $(r=.24$ y $r=$ .22, respectivamente), al utilizar un grupo conformado por estudiantes universitarios y los padres de éstos (n = 573; 123 estudiantes de sexo masculino, 125 estudiantes de sexo femenino, 162 padres y 163 madres). En una réplica y extensión de este trabajo, Greenberg, Lewis y Dodd (1999) hallaron, empleando un grupo de universitarios ( $n=129$; 64 varones y 65 mujeres), que 
la adicción al alcohol se asocia de modo elevado a la adicción al tabaco $(r=.62)$ y moderadamente con la adicción al juego $(r=.38)$.

Desde otro ángulo de análisis, y examinando también el papel de drogas distintas al alcohol, los estudios de comorbilidad sugieren la existencia de vinculaciones entre juego patológico, alcoholismo y consumo de sustancias psicoactivas. Por ejemplo, se ha encontrado que el $47 \%$ de los jugadores patológicos incluidos en un grupo de tratamiento $(n=51)$ también abusaban del alcohol y las drogas en algún momento de sus vidas (Ramírez, McCormick, Russo y Taber, 1983). De modo complementario, Lesieur, Blume y Zoppa (1986) descubrieron, al examinar una amplia muestra de pacientes en tratamiento a causa de sus problemas con el alcohol y las drogas ( $n=458$ ), que un $8 \%$ de los que abusaban del alcohol eran jugadores patológicos, y también lo eran el 18\% de los que abusaban de heroína, el 14\% de los que abusaban de marihuana o el 13\% de los que abusaban de cocaína.

En cuanto a la compra adictiva, nuestros resultados siguen la misma tendencia que los encontrados por Roberts y Tanner (1998) con adolescentes de la población general: se constata que la adicción a la compra covaría con las conductas excesivas consideradas. Sin embargo, la relación de esta adicción conductual con otras adicciones es diferente en la investigación desarrollada por Haylett, Stephenson y Lefever (2004). Estos autores encuentran, al aplicar el Shorter PROMIS Questionnaire (SPQ; Christo, Jones, Haylett, Lefever y Lefever, 2003) a un grupo de adictos a sustancias y actividades en tratamiento ( $n=543$ ), que la adicción a la compra se asocia con diversas adicciones conductuales -las referidas a trabajo, ejercicio, relaciones de ayuda compulsiva, así como a los desórdenes alimentarios-, pero se aleja de un cluster que incluye las adicciones químicas "clásicas" (es decir, las referidas a alcohol, tabaco y drogas) junto a juego, sexo y relaciones de sumisión compulsiva. Por consiguiente, el estudio de Haylett et al. (2004) establece, a diferencia del presente trabajo, una nítida separación entre adicción a la compra y "excesos" en el juego y en el consumo de alcohol y drogas. Previsiblemente, el tipo de muestra, "apetitos excesivos" y/o medidas empleadas podrían explicar las diferentes "vinculaciones" de la compra adictiva encontradas en esta investigación. Obviamente, estos y otros factores condicionan las asociaciones entre conductas adictivas. De hecho, Haylett y sus colegas señalan que la literatura clínica sugiere que las adicciones co-ocurren en conglomerados aparentemente contradictorios a los hallados en su trabajo. Por lo que respecta a la compra adictiva, sirva de ejemplo que varios estudios de comorbilidad han documentado la existencia de porcentajes elevados de compradores adictos con trastornos por consumo de alcohol y drogas (p. ej., Black et al., 1998; McElroy et al., 1994), así como con problemas de juego (p. ej.,
Christenson et al., 1994; Schlosser et al., 1994). En cualquier caso, los hallazgos de nuestro estudio evidencian, empleando una muestra de adultos de la población general, la "conexión" entre adicción a la compra y otras conductas adictivas y apoyan, asimismo, la tesis de comunalidad entre adicciones químicas y conductuales esgrimida por múltiples autores (p. ej., Marks, 1990; Orford, 2001; Peele, 1985, Pomerlau y Pomerlau, 1987; Ridruejo, 1994).

Por último, los hallazgos referidos a las diferencias entre sexos con respecto a los diferentes excesos coinciden con los encontrados en investigaciones previas. Así, son diversos los trabajos que evidencian la superior "inclinación" de las mujeres a la compra adictiva (p. ej., d'Astous, 1990; Friese, 2000; Reisch, 2001; Roberts, 1998), así como los que atestiguan la mayor proclividad de los varones al juego patológico (p. ej., APA, 1994; Becoña, 1993; Ladoucer, 1991; Volberg, 1990), alcoholismo (p. ej., APA, 1994; Bloomfield y Reese, 2000; Chan, Pristach y Welte, 1994; Xunta de Galicia, 2000) y consumo de la mayoría de las drogas ilegales (p. ej., APA, 1994; Grant, 1996; Xunta de Galicia, 2000).

\section{Limitaciones}

Los hallazgos de esta investigación están sujetos a varias limitaciones. Una de éstas es de índole muestral y alude al porcentaje de rechazos a la hora de cumplimentar los cuestionarios (alrededor del 28\%), lo cual no permite garantizar la representatividad poblacional de la muestra empleada (adultos de las capitales gallegas). No obstante, el elevado número de participantes permite, a nuestro juicio, una aproximación aceptable a la materia de estudio en la población de referencia. Un problema adicional es el relativo a la naturaleza transversal del trabajo, que impide analizar aspectos tan valiosos como la posible secuencia temporal de desarrollo de los distintos "excesos"; además, puede dar lugar a resultados erróneos al no considerar cuestiones como el potencial declive de los comportamientos adictivos con la edad o las diferencias generacionales en cuanto a comportamientos excesivos, por ejemplo. La utilización de muestras representativas de una o diversas generaciones y la realización de estudios longitudinales permitirán paliar estos inconvenientes. Una última dificultad de este trabajo es la relativa a las cuestiones de medida. La escala utilizada para evaluar la compra adictiva utiliza ítems que miden, en nuestra opinión, la sensación o percepción de que se compra excesivamente. Por lo tanto, consideramos que sería deseable contar con una medida de adicción a la compra que se base no sólo en sensaciones o percepciones de compra adictiva, sino también en indicadores objetivos de esta conducta adictiva (como el porcentaje de endeudamiento 
o la proporción de ingresos dedicados a compras superfluas, por ejemplo). A pesar de estas dificultades recurrimos al $\mathrm{GABI}$ por ser un cuestionario validado que posee propiedades psicométricas aceptables (unidimensionalidad y elevada consistencia interna) y proporciona, a nuestro juicio, la cobertura conceptual más amplia de la adicción a la compra de entre las medidas actualmente disponibles. La evaluación de los excesos en el juego, comida, tabaco, consumo problemático de alcohol y consumo de sustancias psicoactivas tiene un claro inconveniente: se realiza a partir de un único ítem que mide la frecuencia percibida del comportamiento en cuestión, mientras que sería deseable, como recomienda la ortodoxia científica, emplear medidas validadas y objetivamente verificables de las diferentes adicciones. En cualquier caso, y pese a estos inconvenientes, consideramos que las tendencias constatadas en nuestros hallazgos pueden resultar de interés para los estudiosos de los comportamientos adictivos, si bien será necesario corroborarlas recurriendo a medidas más rigurosas.

\section{Sugerencias para la investigación futura}

Los resultados de la presente investigación parecen apoyar una perspectiva que ha adquirido un creciente protagonismo en el campo de las adicciones durante los últimos años: la que preconiza la inclusión de las adicciones psicológicas, conductuales o no-químicas (según la etiqueta que prefiera utilizarse) en el espectro adictivo. En este sentido, proporcionan apoyo empírico a los planteamientos teóricos según los que los objetos y actividades tienen, al igual que las drogas, la potencialidad de desencadenar adicciones (Orford, 2001). Sin embargo, todavía son pocos los trabajos que han contrastado empíricamente la hipótesis de relación entre adicciones químicas y conductuales. Además, se necesitan desarrollar investigaciones longitudinales que analicen la secuencia evolutiva de las conductas excesivas, incluyendo no sólo drogas legales e ilegales y conductas antinormativas clásicas (agresión, vandalismo, robo, destrucción de la propiedad...) sino, también, los excesos en otros comportamientos (juego, ejercicio, compras, TV, internet, videojuegos, etc.). Un testimonio de la potencial vinculación entre adicciones químicas y conductuales lo proporciona Hirschman (1992), quien refleja, recurriendo a su propia experiencia, un patrón adictivo en el que se simultanea el consumo de estimulantes y alcohol con rituales y atracones de comida y compras.

El estudio de las similitudes entre adicciones "con" y "sin" droga también resulta de interés desde la perspectiva tradicional de la personalidad adictiva. Según diversos autores (p. ej., Greenberg et al., 1999; Rozin y Stoess, 1993), esta controvertida materia, la cual no cuenta con un firme soporte empírico en las investigaciones clásicas realizadas en muestras de alcohólicos y drogodependientes en tratamiento, sería más adecuadamente enfocada si se examinase, como paso previo, la covariación entre adicciones químicas y conductuales en muestras de la población general. La ratificación de estas relaciones permitiría avanzar hacia un "peldaño" superior en la investigación, que sería el referido a analizar las características de personalidad que incrementan la vulnerabilidad hacia los "excesos" múltiples.

Tanto la realización de trabajos encaminados a estudiar la "escalada adictiva", como a desvelar las cualidades de personalidad que aumentan el "riesgo" de multiadicción -o a analizar otras facetas compartidas entre adicciones a sustancias y a actividades ( $p$. ej., genéticas, neurobiológicas, sociales, familiares, del grupo de iguales y contextuales)- contribuirán, a nuestro juicio, no sólo a una mejor comprensión del fenómeno adictivo per se sino, también, a prevenir el desarrollo de pautas adictivas, mejorar la eficacia del tratamiento y evitar/reducir fenómenos como el "salto" entre adicciones o el establecimiento de la denominada "web" de dependencias (Gossop, 2001), los cuales son aspectos que dificultan, en gran medida, el proceso de rehabilitación e integración social del adicto.

\section{REFERENCIAS}

American Psychiatric Association (APA) (1994). Diagnostic and Statistical Manual of Mental Disorders (DSMIV). Washington, DC: The American Psychiatric Association.

Becoña, E. (1993). El juego compulsivo en la Comunidad Autónoma Gallega. Betanzos (A Coruña): Xunta de Galicia.

Black, D. W., Repertinger, M. D., Gaffney, G. R. y Gabel, J. (1998). Family history and psychiatric comorbidity in persons with compulsive buying: Preliminary findings. American Journal of Psychiatry, 155, 960-963.

Bloomfield, K. L. y Reese, A. R. (2000). Prevalence of alcohol use and the association between onset of use and alcohol-related problems in a general population sample in Germany. Addiction, 95, 1389-1401.

Briney, A. L. (1989). An examination of the nature of a problematic consumer behavior: Compulsive purchasing as a learned adaptive, addiction, and personality disorder. Tesis doctoral. Denton, Texas: University of North Texas.

Chan, A. W., Pristach, E. A. y Welte, J. W. (1994). Detection of alcoholism in three populations by the brief-MAST. Alcohol Clinical and Experimental Research, 18, 695701. 
Christenson, G. A., Faber, R. J., de Zwann, M., Raymond, N. C., Specker, S. M., Ekern, M. D., Mackenzie, T. B., Crosby, R. D., Crow, S. J., Eckert, E. D., Mussell, M. P. y Mitchell, J. E. (1994). Compulsive buying: Descriptive characteristics and psychiatric comorbidity. Journal of Clinical Psychiatry, 55, 5-11.

Christo, G., Jones, S. L., Haylett, S., Stephenson, G. M., Lefever, R. M. H. y Lefever, R. (2003). The Shorter PROMIS Questionnaire. Further validation of a tool for simultaneous assessment of multiple addictive behaviors. Addictive Behaviors, 28, 225-248.

Cole, L. K. (1995). Modeling the antecedents of compulsive buying. Tesis doctoral. Louisiana: Louisiana State University.

D'Astous, A. (1990). An inquiry into the compulsive side of "normal" consumers. Journal of Consumer Policy, 13, 15-31.

D'Astous, A., Maltais, J. y Roberge, C. (1990). Compulsive buying tendencies of adolescent consumers. En: M. E., Goldberg, G. Gorn y R. W., Pollay (eds.), Advances in Consumer Research, 17, 396-312. Provo, UT: Association for Consumer Research.

Edwards, E. A. (1992). The measurement and modeling of compulsive consumer buying behavior. Tesis doctoral. Michigan: University of Michigan.

Elliot, R. (1994). Addictive consumption: Function and fragmentation in postmodernity. Journal of Consumer Policy, 17, 159-179.

Faber, R. J., Christenson, G. A., de Zwann, M. y Mitchell, J. (1995). Two forms of compulsive consumption: Comorbidity of compulsive buying and binge eating. Journal of Consumer Research, 22, 296-304.

Faber, R. J. y O'Guinn, T. C. (1989). Classifying compulsive consumers: Advances in the development of a diagnostic tool. En: T. K. Skrull (ed.), Advances in Consumer Research, 16, 738-744. Provo, UT: Association for Consumer Research.

Faber, R. J. y O'Guinn, T. C. (1992). A clinical screener for compulsive buying. Journal of Consumer Research, 19, 459-469.

Faber, R. J., O'Guinn, T. C. y Krych, R. (1987). Compulsive consumption. En: M. Wallendorf y P. Anderson (eds.), Advances in Consumer Research, 14, 132-135. Provo, UT: Association of Consumer Research.

Friese, S. (2000). Self-concept and identity in a consumer society. Aspects of symbolic product meaning. Marburg: Tectum Verlag.

Glatt, M. M. y Cook, C. C. H. (1987). Pathological spending as a form of psychological dependence. British Journal of Addiction, 82, 1257-1258.

Gossop, M. (2001). A web of dependence (Editorial). Addiction, 96, 677-678.

Grant, B. F. (1996). Prevalence and correlates of drug use and DSM-IV drug dependence in the United States: Results of the National Longitudinal Alcohol
Epidemiologic Survey. Journal of Substance Abuse, 8, 195-210.

Greenberg, J. L., Lewis, S. E. y Dodd, D. K. (1999). Overlapping addictions and self-esteem among college men and women. Addictive Behaviors, 24, 565-571.

Haylett, S. A., Stephenson, G. M. y Lefever, R. M. H. (2004). Covariation in addictive behaviors: A study of addictive orientations using the Shorter PROMIS Questionnaire. Addictive Behaviors, 29, 61-71.

Hirschman, E. C. (1992). The consciousness of addiction: Toward a general theory of compulsive consumption. Journal of Consumer Research, 19, 155-179.

King, A. D. (1981). Beyond propensities: Toward a theory of addictive consumption. En K. Bernhardt et al. (eds.), The changing marketing environment: New theories and applications (pp. 438-440). Chicago, IL: American Marketing Association.

Ladoucer, R. (1991). Prevalence estimates of pathological gamblers in Quebec, Canada. Canadian Journal of Psychiatry, 36, 732-734.

Lesieur, H. R., Blume, S. B. y Zoppa, R. M. (1986). Alcoholism, drug abuse, and gambling. Alcoholism: Clinical and Experimental Research, 10, 33-38.

Luna, R. (2002). Segmentos de consumidores según la escala de compra patológica. Investigación y Marketing, $77,16-25$.

Marks, I. (1990). Behavioural (non chemical) addictions. British Journal of Addictions, 85, 1389-1394.

McElroy, S. L., Keck, P. E. Jr., Pope, H. G. Jr., Smith, J. M. R. y Strakowski, S. M. (1994). Compulsive buying: A report of 20 cases. Journal of Clinical Psychiatry, 55, 242-248.

Mowen, J. C. y Spears, N. (1999). Understanding compulsive buying among college students: A hierarchical approach. Journal of Consumer Psychology, 8, 407430.

Nataraajan, R. y Goff, B. G. (1991). Compulsive buying: Towards a reconceptualization. Journal of Social Behavior and Personality, 6, 307-328.

O'Guinn, T. C. y Faber, R. J. (1989). Compulsive buying: A phenomenological exploration. Journal of Consumer Research, 16, 147-157.

Olábarri, E. y García, I. (2003). La compra por impulso y la adicción al consumo en el País Vasco. Estudios sobre Consumo, 65, 53-67.

Orford, J. (2001). Excessive appetites: A psychological view of addictions. Chichester: John Wiley.

Peele, S. (1985). The meaning of addiction. A compulsive experience and its interpretation. Lexington, MA: Lexington books.

Pomerlau, O. F. y Pomerlau, C. S. (1987). A biobehavioral view of substance abuse and addiction. Journal of Drug Issues, 17, 111-132. 
Ramírez, L. F., McCormick, R. A., Russo, A. M. y Taber, J. L. (1983). Patterns of substance abuse in pathological gamblers undergoing treatment. Addictive Behaviors, 8, 425-428.

Reisch, L. A. (2001). Women and addictive buying: The gender question revised. En: I. García y E. Olábarri (eds.), El consumo y la adicción a las compras: Diferentes perspectivas (pp. 169-195). Bilbao: Universidad del País Vasco.

Reisch, L. A. y Scherhorn, G. (1996). Women and addictive buying: Theory and Research. Stuttgart: Universität Hohenheim, Institut für Konsumtheorie und Verbraucherpolitik, Arbeitspapier 70.

Ridruejo, P. (1994). Hacia un modelo integral de adicción. En: M. Casas, M. Gutiérrez y L. San (coord.), Psicopatología y alcoholismo (pp. 513-549). Barcelona: Ediciones en Neurociencias.

Roberts, J. A. (1998). Compulsive buying among college students: An investigation of its antecedents, consequences, and implications for public policy. The Journal of Consumer Affairs, 32, 295-319.

Roberts, J. A. y Martínez, C. R. (1997). The emerging consumer culture in Mexico: An exploratory investigation of compulsive buying in Mexican young adults. Journal of International Consumer Marketing, 10, 7-31.

Roberts, J. A. y Tanner, J.R. (2000). Compulsive buying and risky behavior among adolescents. Psychological Reports, 86, 763-770.
Rodríguez-Villarino, R. (2004). Naturaleza y alcance de la adicción a la compra en la población urbana de Galicia: Un estudio empírico. Tesis doctoral. Ourense: Universidad de Vigo.

Rozin, P. y Stoess, C. (1993). Is there a general tendency to become addicted? Addictive Behaviors, 18, 81-87.

Sánchez, S. (1996). Compra compulsiva: Un estudio en depresión, bulimia y controles. Tesis doctoral. Valladolid: Universidad de Valladolid.

Scherhorn, G., Reisch, L. A. y Raab, G. (1990). Addictive buying in West Germany: An empirical study. Journal of Consumer Policy, 13, 355-387.

Schlosser, B. A., Black, D. W., Repertinger, S. y Freet, D. (1994). Compulsive buying: Demography, phenomenology, and comorbidity in 46 subjects. General Hospital Psychiatry, 16, 205-212.

Specker, S. M., Carlson, G. A., Christenson, G. A. Y Marcotte, M. (1995). Impulse control disorders and attention deficit disorder in pathological gamblers. Annals of Clinical Psychiatry, 7, 175-179.

Volberg, R. A. (1990). Estimating the prevalence of pathological gambling in the United States. Artículo presentado a la Eight International Conference on Risk and Gambling, August.

Xunta de Galicia (2000). O consumo de drogas en Galicia V. Santiago de Compostela (A Coruña): Xunta de Galicia. 


\section{Apéndice 1}

German Addictive Buying Indicator (GABI; Scherhorn, Reisch y Raab, 1990)

A continuación aparecen una serie de afirmaciones referidas al comportamiento de compra. Por favor, indica tu nivel de acuerdo con respecto a cada una de ellas y marca con un aspa $(X)$ el número que mejor se adapte a tu caso particular, teniendo en cuenta las siguientes equivalencias:

1 = Totalmente en desacuerdo

2 = En desacuerdo

$3=$ De acuerdo

4 = Totalmente de acuerdo

1. Cuando tengo dinero siento la necesidad de gastarlo.

\begin{tabular}{|c|c|c|}
\hline 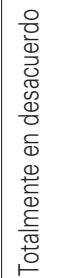 & 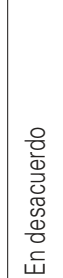 & 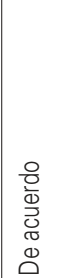 \\
\hline 1 & 2 & 3 \\
\hline
\end{tabular}

2. Cuando paso por el centro de la ciudad o por un centro comercial, a menudo siento la necesidad de comprar.

3. Muchas veces siento unas ganas inexplicables de salir y comprar cosas que deseo de modo urgente y repentino.

4. A veces veo algo y siento un impulso irresistible de comprarlo.

5. Muchas veces tengo la sensación de que tengo que comprar algo determinado.

6. Después de comprarme algo me pregunto muchas veces si realmente era tan importante.

7. Muchas veces compro cosas sólo porque son baratas.

12534

8. Muchas veces compro cosas simplemente porque tengo ganas de comprar.

9. La publicidad me parece muy interesante; a menudo también encargo algo (compra a distancia).

10. Muchas veces he comprado cosas que después no he utilizado.

$\begin{array}{llll}1 & 2 & 3 & 4 \\ -1\end{array}$

11. Muchas veces me he comprado cosas que en realidad no podía permitirme.

12. Soy un derrochador.

$\begin{array}{llll}1 & 2 & 3 & 4\end{array}$

13. Para mí comprar es un modo de escaparme de la rutina de la vida cotidiana y relajarme.

14. A veces noto algo dentro de mí que me empuja a comprar.

15. A veces tengo remordimientos de conciencia después de haberme comprado algo.

16. Muchas veces no me atrevo a enseñar a los demás las cosas que he comprado porque podría parecer una persona irresponsable. 